\title{
Circumscribed choroidal haemangioma: Indocyanine green angiography features on scanning laser ophthalmoscopy versus traditional digital fundus photography.
}

\author{
Kelsey A. Roelofs MD, FRCSC 1,2 \\ David I.T. Sia MBChB, FRANZCO ${ }^{1,2}$ \\ Roderick O’Day MBBS, FRANZCO ${ }^{1,2}$ \\ Gordon Hay, MBBS ${ }^{1,2}$ \\ Amit K. Arora MBBS, MRCOphth 1,2 \\ Victoria M.L. Cohen FRCOphth ${ }^{1,2}$ \\ Mandeep S. Sagoo MB, PhD, FRCOphth ${ }^{1,2}$
}

${ }^{1}$ Ocular Oncology Service, Moorfields Eye Hospital, London, United Kingdom

${ }^{2}$ NIHR Biomedical Research Centre for Ophthalmology at Moorfields Eye Hospital and University College London Institute of Ophthalmology.

Running title: ICG features of circumscribed choroidal haemangioma

\section{Corresponding Author:}

Professor Mandeep Sagoo

Moorfields Eye Hospital NHS Foundation Trust, 162 City Road, London, EC1V 2PD

Tel: 02072533411

\section{Email:m.sagoo@ucl.ac.uk}

No financial support was received for this research. None of the authors have any financial dislcosures or conflicts of interest to declare. This manuscript has not previously been submitted for publication and has not been presented at a meeting. 
Abstract Word Count: 236 words

Manuscript Word Count: 2538

Keywords: Circumscribed choroidal haemangioma, choroidal tumour, indocyanine green angiography, Heidelberg Spectralis, Topcon, fundus imaging, scanning laser ophthalmoscopy 


\section{ABSTRACT:}

Background and Objective: Circumscribed choroidal haemangioma $(\mathrm{CCH})$ has several characteristic clinical and angiographic features. We aimed to compare indocyanine green angiography (ICG) findings of $\mathrm{CCH}$ captured on a traditional digital camera system (DCS) to newer scanning laser ophthalmoscopy (SLO) platforms.

Study Design/Materials and Methods: A total of 35 patients over a 10 year period diagnosed with CCH using ICG were included (18 imaged with DCS and 17 with SLO).

Results: On early ICG frames, intrinsic vessels were apparent in two thirds $(12 / 18 ; 67 \%)$ of the DCS group compared to all of eyes in the SLO group $(\mathrm{p}=0.020)$. Additionally, at maximal hyperfluorescence, most eyes imaged with DCS had a feathery appearance $(16 / 18 ; 89 \%)$ compared to those in the SLO group which all $(17 / 17 ; 100 \%)$ displayed a granular appearance ( $p$ $<0.001)$. The presence of hot spots at maximal hyperfluorescence was also more common in the SLO group $(12 / 17 ; 71 \%)$ versus the DCS group $(0 / 18 ; 0 \%)(p<0.001)$. Finally, intrinsic vessels and vascular loops could be identified throughout the entire duration of the ICG in $100 \%$ of the SLO cases $(17 / 17)$ versus only $11 \%(2 / 18)$ of DCS cases $(p<0.001)$.

Conclusion: The visualization of intrinsic vessels, vascular loops and "hot spots" in $\mathrm{CCH}$ is significantly enhanced with SLO compared to DCS. Many characteristic mid-late angiographic findings of $\mathrm{CCH}$ are more optimally visualized on SLO which may negate the need for late frames (>30 minutes) without compromising diagnostic accuracy. 


\section{BACKGROUND and OBJECTIVE:}

Circumscribed choroidal haemangiomas $(\mathrm{CCH})$ are benign, vascular tumours that tend to be solitary and unilateral in nature. They have a characteristic red-orange appearance and can have overlying RPE hyperplasia (33\%) or fibrous metaplasia (20\%). ${ }^{1}$ Most commonly, $\mathrm{CCH}$ involve the superotemporal quadrant of the macula ${ }^{2}$ and tend to present in the $5^{\text {th }}$ decade of life, often with decreased visual acuity and a serous macular detachment. ${ }^{1}$ Circumscribed choroidal haemangioma has historically been categorized as a congenital lesion without associated ocular or systemic anomalies. ${ }^{3}$ However, recent reports of increased sub-foveal choroidal thickness ${ }^{4}$ and irregular choroidal vascular architecture have suggested that predisposing ocular factors may impart a propensity for the growth of this hamartomatous lesion. ${ }^{5}$

Several findings on ancillary studies can support the diagnosis of $\mathrm{CCH}$. Clinically, $\mathrm{CCH}$ often transilluminates during slit lamp biomicroscopy when a small, bright beam is centred over the lesion. This vascular tumour shows high internal reflectivity on ultrasound A-scan and appears as a solid, dome shaped mass on ultrasound B-scan. ${ }^{1,6}$ Enhanced depth imaging optical coherence tomography (EDI-OCT) shows expansion of vessels in Haller's and Sattler's layers, ${ }^{7}$ but unlike choroidal nevi, ${ }^{8}$ melanoma ${ }^{9}$ and metastasis,${ }^{10}$ the choriocapillaris is not compressed. On fluorescein angiography (FA), progressive hyperfluorescence is typically seen with diffuse, late staining of the tumour; ${ }^{1,2}$ however, given that these features may be absent in up to two thirds of cases, many consider indocyanine green angiography (ICG) to supersede FA.

Given the anatomical location of $\mathrm{CCH}$, indocyanine green angiography (ICG) has been employed with a characteristic pattern of early uptake by the tumour, followed by an increase in fluorescent intensity, with late washout due to its highly vascular nature. Previously published landmark studies describing ICG characteristics of $\mathrm{CCH}$ used digital fundus photography based imaging systems (Topcon TRC 50 IA fundus camera). ${ }^{11}$ The purpose of this study was to compare the well documented colour fundus photographic and ICG characteristics of $\mathrm{CCH}$ captured on digital camera systems (DCS) to the features appreciated on newer scanning laser ophthalmoscopy (SLO) platforms (Optos California and Heidelberg Spectralis).

\section{PATIENTS/MATERIALS and METHODS:}

Following registration with and approval by the clinical audit department (audit number 530), consecutive eyes diagnosed with $\mathrm{CCH}$ over a ten year period (01/01/2007 to 31/12/2017) in the Ocular Oncology Service of Moorfields Eye Hospital were identified. In 2016 we began transitioning from ICG on DCS to SLO. A total of 35 eyes that had ICG studies taken on standard imaging settings were included; 18 of these had digital ICG photographs taken using DCS (Topcon fundus camera, Topcon Corporation, Tokyo, Japan) and 17 had images captured using SLO platforms (Optos California [Optos plc, Dunfermline, Scotland] and Heidelberg Spectralis [Heidelberg Engineering GmbH, Heidelberg, Germany]). All of those imaged with 
SLO had ICG angiography captured on Heidelberg Spectralis and fundus photographs taken with Optos.

The DCS used a halogen lamp, a polychromatic light source, to illuminate the retina. For ICG, an excitation filter of $640-780 \mathrm{~nm}$ and a barrier filter of $820-900$ was used, respectively.

In the SLO group a monochromatic laser light source is used to illuminate the retina, rather than a halogen lamp as is used in DCS. The Optos California images were obtained using a combination of four laser wavelengths: blue $(488 \mathrm{~nm})$, green $(532 \mathrm{~nm})$, red $(635 \mathrm{~nm})$ and infrared $(802 \mathrm{~nm})$. The Optomap ${ }^{\circledR}$ pseudo-colour image was generated using the green and red lasers, whereas the Optos autofluorescence image was generated using the green laser. The Heidelberg Spectralis OCT used a wavelength of 870nm and the Spectralis ICG excitation was by a diode laser of $790 \mathrm{~nm}$ and a barrier filter of $830 \mathrm{~nm} .^{12}$

Patient files were reviewed for demographic data, including gender and age at time of diagnosis. All images were reviewed and variables recorded included colour of the tumour on DCS versus SLO as well as ICG features of the tumour, such as visualization of intrinsic vessels and presence or absence of "hot spots". The pattern at maximal hyperfluorescence was categorized either 'feathery' or 'granular'. Lesions categorized as 'feathery' had blurred tumour margins and indistinct intra-lesional features. Those defined as 'granular' had distinct margins and pixelated intra-tumoral characteristics. Diffuse versus patchy were used as additional descriptive terms, with the former indicating a homogenous appearance throughout the entire lesion. As there was variability amongst cases with respect to duration of imaging, the timing of the latest frame was also recorded. This image was assessed for a relative decrease in fluorescence of the lesion, presence of "hot spots", "washout" of the lesion and presence of a hyperfluorescent rim. "Washout" was further classified as complete versus partial depending on whether the entire lesion or only a portion of it became hypofluorescent compared to the rest of the choroid. We defined 'early' as $<1$ minute, 'mid' as 1-3 minutes and 'late' as $>7$ minutes.

Data are presented as mean \pm standard deviation (SD) when normally distributed or as median and [interquartile range] if not. Normality was assessed using the Shapiro-Wilks test. Differences in continuous variables were compared using a student's t-test or the Mann-Whitney U test for normally and not normally distributed data, respectively. Differences in proportions between the groups were analysed using Fisher's exact test with the Freeman-Halton extension. A $P$-value of $<0.05$ was considered statistically significant. All data were analyzed using a commercially available software package (SPSS ${ }^{\circledR} 25$; IBM Corporation, Armonk, NY, USA).

\section{RESULTS:}

A total of 35 eyes with $\mathrm{CCH}$ evaluated with ICG angiography were included in this study. The mean age at diagnosis for the entire cohort was $53.8 \pm 13.2$ years. The majority of 
patients were male (74\%) and right eyes (63\%) were affected more commonly than left. Approximately half of the cases had imaging captured on DCS (18 eyes, 51\%) versus SLO (17 eyes, 49\%). Overall, the mean ( \pm SD) $\mathrm{CCH}$ thickness and largest basal diameter (LBD) was 2.1 $\pm 1.8 \mathrm{~mm}$ and $6.8 \pm 1.9 \mathrm{~mm}$. Most lesions were associated with sub-retinal fluid $(24 / 27 ; 89 \%)$ and located within the macula $(25 / 35 ; 71 \%)$. There was no difference $\mathrm{CCH}$ characteristics between the DCS and SLO groups. (Table 1).

The appearance of $\mathrm{CCH}$ on the colour image varied significantly between the groups. Images captured with DCS more accurately represented the true red-orange fundoscopic appearance of $\mathrm{CCH}$ (Figure 1A and 2A) compared to SLO, where the lesion appeared to have a green-grey hue (Figure 3A and 4A). As SLO produces an artificial colour image created by the reflectance patterns of two laser wavelengths, the red and green images can be separated using the blend function. In cases of $\mathrm{CCH}$, the red separation image (Figure 3B and 4B) further highlights the choroidal location and true extent of this lesion, compared to the green separation image (Figure 3C and 4C).

During ICG angiography, several notable differences were seen between the two groups. On early frames, intrinsic vessels were apparent in two thirds $(12 / 18 ; 67 \%)$ of the DCS group compared to all of eyes in the SLO group $(\mathrm{p}=0.020)$. At maximal hyperfluorescence, most eyes imaged with DCS had a feathery appearance (16/18; 89\%) (Figures 1C and 2C) compared to those in the SLO group which all displayed a granular appearance (Figures 3E and 4E) (p $<0.001)$. Of the 16 eyes demonstrating a feathery appearance on DCS, $22 \%(4 / 16)$ showed patchy variation throughout the lesion and 67\% (12/16) had a diffuse homogenous appearance. The presence of hot spots at maximal hyperfluorescence reached a statistically significant difference between the modalities, as hot spots were apparent in the majority of SLO (12/17; $71 \%)$ versus none of the DCS cases $(0 / 18 ; 0 \%)(p<0.001)$.

There was no difference in the latest frame recorded between the two imaging modalities $(p=0.590)$ and irrespective of the imaging platform employed, all eyes showed a relative decrease in fluorescence in the late frames. More eyes imaged on SLO displayed a complete $(3 / 17 ; 18 \%)$ or partial $(10 / 17 ; 59 \%)$ washout compared to DCS $(1 / 18 ; 6 \%$ and $6 / 18 ; 33 \%$, respectively), though this effect did not reach statistical significance $(\mathrm{p}=0.076)$. Significantly more eyes in the SLO cohort displayed a late hyperfluoresent rim (11/17; 65\% versus 4/18; 22\%; $\mathrm{p}=0.043$ ) and hot spots were more commonly a prominent feature of the late frames captured on SLO $(14 / 17 ; 82 \%)$ compared to DCS $(5 / 18 ; 28 \%)(p=0.003)$.

The most striking difference between the imaging modalities was in the ability to identify intrinsic vessels and vascular loops throughout the entire duration of the ICG $(2 / 18 ; 11 \%$ of DCS cases versus $17 / 17 ; 100 \%$ of SLO cases) $(\mathrm{p}<0.001)($ Table 1$)$.

\section{DISCUSSION:}


Differentiation of non-pigmented choroidal lesions based solely upon their clinical appearance can be challenging, and as such, multi-modal imaging plays a crucial role in narrowing the diagnostic considerations. Although several features of $\mathrm{CCH}$ have been well described, in two large series only $1 / 3^{\text {rd }}$ of patients were referred with the correct diagnosis. ${ }^{1,2}$ Circumscribed choroidal haemangiomas tend to have a solid, dome shape appearance with relatively high internal reflectivity on ultrasonography ${ }^{1,6}$ and a smooth, gently sloping contour without choriocapillaris compression on EDI-OCT. ${ }^{7}$ Their typical red-orange colour leads to a wide differential diagnosis, which can be refined by the use of multi-modal imaging.

Yannuzzi et al first described ICG angiography with DCS as a tool to enhance choroidal imaging and in particular, to assist in the detection of occult choroidal neovascular membranes in patients with age-related macular degeneration (AMD). ${ }^{13}$ In contemporary clinical practice, ICG is used to aide in the diagnosis, classification and treatment of a select group of chorioretinal disorders, ${ }^{14}$ including polypoidal choroidal vasculopathy ${ }^{15}$ and retinal angiomatous proliferation, ${ }^{16}$ central serous chorioretinopathy, ${ }^{17,18}$ choroidal inflammatory conditions, ${ }^{19}$ and $\mathrm{CCH} \cdot{ }^{11,20,21}$

On ICG captured with DCS, CCH display early hyperfluorescence, late "washout", visualization of intrinsic vessels, a late hyperfluorescent rim and late "hot spots". ${ }^{11}$ However, as patterns on ICG classically reported using DCS differ from that observed with newer SLO platforms, there is a need to further describe the latter. Compared to previous studies, our series had a relatively low rate of "washout" in both groups. This is likely due to the fact that only three patients had 'late' frames acquired $>30$ minutes. However, the characteristic 'early' and 'mid' frame findings reported in this series suggest that in some cases, acquisition of images $>30$ minutes are not required to confirm the diagnosis.

In a large series, $29 \%$ of patients with $\mathrm{CCH}$ were referred with the diagnosis of choroidal melanoma ${ }^{1}$ and overall, $\mathrm{CCH}$ comprises roughly $5 \%$ of all cases of pseudomelanomas. ${ }^{22}$ Several ancillary studies may aide in differentiating the former from the latter, including ultrasonography with standardized A-scan (high versus low internal reflectivity) and sparing versus compression of the choriocapillaris on EDI-OCT. ${ }^{9}$ Moreover, on ICG choroidal melanomas reach maximum hyperfluorescence much later than $\mathrm{CCH}$ (18.2 minutes versus 1.2 minutes; respectively) and the majority of choroidal melanomas ${ }^{23}$ and choroidal nevi remain hypofluorescent compared to the surrounding choroid throughout the entire angiographic study. ${ }^{24}$

\section{Appearance of $\mathrm{CCH}$ on colour photos and red versus green separation images}

Circumscribed choroidal haemangioma clinically appears as a red-orange choroidal mass, most commonly in the superotemporal quadrant near the macula. ${ }^{2}$ The Optomap ${ }^{\circledR}$ image is artificially constructed from the reflectance patterns of two laser wavelengths, which have variable penetrance of the retinal structure. The green laser is unable to penetrate the retinal pigment epithelium (RPE), whereas the red is readily able to. Consequently, these images can be 
useful in giving information as to the depth of a lesion. Grey-green lesions on pseudo-colour SLO imaging tend to be choroidal. ${ }^{25}$ However, the darker pseudo-colour of $\mathrm{CCH}$ may further simulate the appearance of pigmented lesions, such as choroidal nevi or melanoma. As such, it is critical for clinicians to be aware of the mis-leading colour photographic representation of $\mathrm{CCH}$ on SLO to avoid this diagnostic pitfall. Although the pseudo-colour image of SLO less accurately reflects the true fundoscopic appearance, its fundamental constructs can be exploited to produce a red separation image that more clearly highlights the true extent of the $\mathrm{CCH}$, which may be underestimated on DCS.

\section{Presence of intrinsic vessels}

Balasubramaniam et al aimed to characterize the features of choroidal tumours on infrared (IR) imaging, and found that vascular loops on IR imaging showed a high diagnostic sensitivity for $\mathrm{CCH}^{20}$ Similarly, we found that all patients undergoing ICG imaging with SLO platforms had vascular loops and intrinsic vessels visualized throughout the entire angiogram. While DCS captured intrinsic vessels in two-thirds of patients in early frames, the visualization of this feature was only apparent in $11 \%(2 / 18)$ of patients throughout the entire angiogram.

\section{Pattern in mid and late frames}

At maximal hyperfluorescence, all eyes imaged in the SLO group had a granular appearance, again with visualization of intrinsic vessels and vascular loops. This appearance was markedly different from the feathery, diffuse and rather homogenous hyperfluorescence seen in the DCS group. On late frames, hot spots were present in 100\% in the SLO group versus $67 \%$ of the DCS group. Moreover, the "hot spots" were subjectively more intense in the SLO group compared with the DCS group. Arevalo et al hypothesized that the "hot spots" visualized on late frames represent residual dye remaining in the intrinsic vessels of $\mathrm{CCH} .{ }^{11}$ Taken together, the findings of this series suggest that intrinsic vessels are better imaged with SLO, which may explain why late "hot spots" were also a consistently prominent finding.

\section{The role of ICG in the setting of multi-modal imaging}

When evaluating a non-pigmented choroidal lesion, ICG findings should not be interpreted in isolation, but rather in consort with the findings of other non-invasive imaging modalities, including ultrasonography with A- and B-scans and EDI-OCT. An experienced clinician may not find angiographic studies essential to confirm the diagnosis of $\mathrm{CCH}$ in classic cases; however, relying on ophthalmoscopic appearance alone is fraught with pitfalls and may

lead to misdiagnosis of malignant lesions as $\mathrm{CCH}$, resulting in a delay of appropriate treatment. ${ }^{26}$

The differential diagnosis of red-orange fundus lesions includes both benign and malignant tumours. Choroidal metastasis, particularly those arising from bronchial carcinoid tumour, thyroid cancer or renal cell carcinoma, can mimic the red-orange appearance of $\mathrm{CCH}$. Enhanced depth imaging-OCT may be particularly illuminating in these cases, as choroidal 
metastasis often show a characteristic "lumpy bumpy" topography with compression of the overlying choriocapillaris, ${ }^{27}$ and on ICG a subtle, diffuse homogenous isofluorescence with late hypofluorescence is often seen. ${ }^{23}$ Uveal lymphoma may also mimic $\mathrm{CCH}$; however, hypofluorescence throughout $\mathrm{ICG}^{28}$ and a "flat, rippled, or undulating (seasick)" topography on EDI-OCT ${ }^{29}$ help to distinguish this entity.

As technology continues to advance in the field of ophthalmic imaging, novel modalities, such as OCT-angiography (OCT-A) may supplant traditional angiography in certain chorioretinal conditions. However, the current utility of OCT-A in CCH is limited given the inability of this technology to capture vascular anatomy deep to the superficial choriocapillaris. Given that the choriocapillaris is uninvolved in $\mathrm{CCH}^{30}$ ICG remains the only angiographic modality by which to assess the affected Haller's and Sattler's layers in this entity.

\section{The role of ICG in guiding treatment of chorioretinal disorders including $\mathrm{CCH}$}

In addition to aiding in the accurate diagnosis of chorioretinal conditions, ICG has be used to guide laser photocoagulation of occult choroidal neovascularization ${ }^{31}$ and photodynamic therapy for central serous chorioretinopathy. ${ }^{18}$ Although several treatment modalities for $\mathrm{CCH}$ exist, including argon laser photocoagulation, transpupillary thermotherapy, plaque brachytherapy and lens sparing external beam radiotherapy, recent reports have shown excellent visual outcomes following treatment with PDT. ${ }^{32,33}$ Indocyanine green guided PDT allows for more precise spot size selection and placement, thus optimizing treatment of the $\mathrm{CCH}$ while minimizing unnecessary damage to surrounding structures.

Limitations of this report relate primarily to the duration of ICG angiography studies. The mean latest frame for all 35 patients included occurred at $13 \mathrm{~min} 13 \mathrm{sec}$, which is a significantly shorter duration than previous reports that recorded the latest frame at $35 \mathrm{~min}^{.1}$ While the difference in angiography times between groups was not statistically significant, only three patients in this study had late frames $>30$ minutes and all three were in the SLO group. As a result, we have emphasized the early and mid-frame findings of $\mathrm{CCH}$ in this report, but noteworthy is that all cases on either imaging platform showed a decrease in fluorescence by the latest frame taken.

This report has updated the ICG imaging characteristics of $\mathrm{CCH}$ using widely available platforms in modern ophthalmic practice that utilise a SLO imaging system. In summary, ICG imaging of $\mathrm{CCH}$ on SLO platforms shows more prominent intrinsic vessels and vascular loops that remain visible throughout the entire angiogram, a granular rather than feathery diffuse hyperfluorescence in mid frames and more conspicuous "hot spots" on mid-late frames compared to DCS. Addition of these early and mid-frame characteristic features to the clinical assessment of suspected $\mathrm{CCH}$ may negate the need for late frames $>30$ minutes without compromising diagnostic accuracy. 
ACKNOWLEDGEMENTS: All authors contributed to the body of work presented herein. The authors would like to acknowledge the Ophthalmic Imaging Department at Moorfields Eye Hospital for their role in image acquisition.

The research was supported by the National Institute for Health Research (NIHR) Biomedical Research Centre based at Moorfields Eye Hospital NHS Foundation Trust and UCL Institute of Ophthalmology. The views expressed are those of the authors and not necessarily those of the NHS, the NIHR or the Department of Health.

CONFLICT OF INTEREST: None of the authors have any financial disclosures or conflicts of interest to declare.

FUNDING: No funding was obtained for this research. 
Table 1. Comparison of demographic and imaging characteristics of patients with circumscribed choroidal hemangioma undergoing ICG imaging with either digital camera system (Topcon) or scanning laser ophthalmoscope (Heidelberg Spectralis)

\begin{tabular}{|c|c|c|c|}
\hline & $\begin{array}{l}\text { DCS } \\
\mathrm{n}=18\end{array}$ & $\begin{array}{l}\text { SLO } \\
\mathbf{n}=17\end{array}$ & p-value \\
\hline Age (mean \pm SD) & $49.9 \pm 12.8$ & $57.9 \pm 12.7$ & 0.071 \\
\hline $\begin{array}{l}\text { Gender }(\%) \\
\quad \text { Male } \\
\text { Female }\end{array}$ & $\begin{array}{l}13(72) \\
5(28)\end{array}$ & $\begin{array}{l}13(76) \\
4(24)\end{array}$ & 1.000 \\
\hline \multicolumn{4}{|l|}{$\begin{array}{l}\text { Hemangioma characteristics } \\
\text { Size (mm) }\end{array}$} \\
\hline Thickness (mean \pm SD) & $2.3 \pm 1.0$ & $2.1 \pm 0.7$ & 0.872 \\
\hline Largest basal dimension (mean $\pm \mathrm{SD})$ & $6.9 \pm 2.3$ & $6.8 \pm 1.4$ & 0.938 \\
\hline Presence of SRF* $(\%)$ & $10 / 10(100)^{*}$ & $14 / 17(82)$ & 0.274 \\
\hline Located within the macula & $11 / 18(61)$ & $14 / 17(82)$ & 0.462 \\
\hline $\begin{array}{l}\text { Intrinsic vessels and vascular loops visible on early } \\
\text { frames }(\%)\end{array}$ & $12 / 18(67)$ & $\begin{array}{c}16 / 16 \\
(100)^{* *}\end{array}$ & $\mathbf{0 . 0 2 0}$ \\
\hline $\begin{array}{l}\text { Pattern at maximal hyperfluorescence }(\%) \\
\text { Granular } \\
\text { Feathery } \\
\text { Patchy } \\
\text { Diffuse }\end{array}$ & $\begin{array}{l}2 / 18(11) \\
16 / 18(89) \\
4 / 16(22) \\
12 / 16(67)\end{array}$ & $\begin{array}{c}17 / 17(100) \\
0 / 17(0)\end{array}$ & $<0.001$ \\
\hline Presence of hot spots at maximal hyperfluorescence $(\%)$ & $0(0)$ & $12(71)$ & $<0.001$ \\
\hline Latest frame recorded (sec) $($ mean \pm SD) & $677 \pm 159$ & $918 \pm 720$ & 0.590 \\
\hline Decrease in fluorescence at latest frame (\%) & 18/18 (100) & $17 / 17(100)$ & 1.000 \\
\hline $\begin{array}{l}\text { Washout at latest frame }(\text { no. cases } / \%) \\
\text { Complete } \\
\text { Partial } \\
\text { No }\end{array}$ & $\begin{array}{c}1(6) \\
6(33) \\
11(61)\end{array}$ & $\begin{array}{l}3(18) \\
10(59) \\
4(24)\end{array}$ & 0.076 \\
\hline $\begin{array}{l}\text { Later hyperfluorescent rim }(\%) \\
\text { Complete } \\
\text { Partial } \\
\text { No }\end{array}$ & $\begin{array}{c}1 / 18(6) \\
3 / 18(17) \\
14 / 18(78)\end{array}$ & $\begin{array}{l}5 / 17(29) \\
6 / 17(35) \\
6 / 17(35)\end{array}$ & 0.043 \\
\hline $\begin{array}{l}\text { Hot spots on late frames } \\
\text { Prominent } \\
\text { Subtle } \\
\text { No }\end{array}$ & $\begin{array}{l}5 / 18(28) \\
7 / 18(39) \\
5 / 18(28)\end{array}$ & $\begin{array}{l}14 / 17(82) \\
3 / 17(18) \\
0 / 17(0)\end{array}$ & 0.003 \\
\hline $\begin{array}{l}\text { Visualization of intrinsic vessels throughout entire ICG } \\
(\%)\end{array}$ & $2 / 18(11)$ & $17 / 17(100)$ & $<0.001$ \\
\hline
\end{tabular}

DCS-digital camera system; SLO-scanning laser ophthalmoscopy.; SRF-sub-retinal fluid

* the presence or absence of sub-retinal fluid was not clearly documented in 8 cases

** early frames were not available for one patient in the SLO group 


\section{REFERENCES:}

1. Shields CL, Honavar SG, Shields JA, Cater J, Demirci H. Circumscribed choroidal hemangioma: clinical manifestations and factors predictive of visual outcome in 200 consecutive cases. Ophthalmology. 2001;108(12):2237-2248.

2. Krohn J, Rishi P, Froystein T, Singh AD. Circumscribed choroidal haemangioma: clinical and topographical features. Br J Ophthalmol. 2019.

3. Singh AD DB, Pe'er J,eds. Clinical ophthalmic oncology. Philadelphia, PA: Saunders Elsevier; 2007.

4. Kim RS, Jain RR, Brown DM, et al. Elevated Choroidal Thickness and Central Serous Chorioretinopathy in the Fellow Eyes of Patients with Circumscribed Choroidal Hemangioma. Ocul Oncol Pathol. 2018;4(6):375-380.

5. Sobol EK, Francis JH, Abramson DH, Freund KB, Spaide RF, Barbazetto I. Subfoveal Choroidal Thickness and Vascular Architecture in Fellow Eyes of Patients with Circumscribed Choroidal Hemangioma. Retina. 2019.

6. Verbeek AM, Koutentakis P, Deutman AF. Circumscribed choroidal hemangioma diagnosed by ultrasonography. A retrospective analysis of 40 cases. Int Ophthalmol. 1995;19(3):185-189.

7. Rojanaporn D, Kaliki S, Ferenczy SR, Shields CL. Enhanced depth imaging optical coherence tomography of circumscribed choroidal hemangioma in 10 consecutive cases. Middle East Afr J Ophthalmol. 2015;22(2):192-197.

8. Shah SU, Kaliki S, Shields CL, Ferenczy SR, Harmon SA, Shields JA. Enhanced depth imaging optical coherence tomography of choroidal nevus in 104 cases. Ophthalmology. 2012;119(5):1066-1072.

9. Shields CL, Kaliki S, Rojanaporn D, Ferenczy SR, Shields JA. Enhanced depth imaging optical coherence tomography of small choroidal melanoma: comparison with choroidal nevus. Arch Ophthalmol. 2012;130(7):850-856.

10. Witkin AJ, Fischer DH, Shields CL, Reichstein D, Shields JA. Enhanced depth imaging spectral-domain optical coherence tomography of a subtle choroidal metastasis. Eye (Lond). 2012;26(12):1598-1599.

11. Arevalo JF, Shields CL, Shields JA, Hykin PG, De Potter P. Circumscribed choroidal hemangioma: characteristic features with indocyanine green videoangiography. Ophthalmology. 2000;107(2):344-350.

12. Hassenstein A, Meyer CH. Clinical use and research applications of Heidelberg retinal angiography and spectral-domain optical coherence tomography - a review. Clin Exp Ophthalmol. 2009;37(1):130-143.

13. Yannuzzi LA, Slakter JS, Sorenson JA, Guyer DR, Orlock DA. Digital indocyanine green videoangiography and choroidal neovascularization. Retina. 1992;12(3):191-223.

14. Yannuzzi LA. Indocyanine green angiography: a perspective on use in the clinical setting. Am J Ophthalmol. 2011;151(5):745-751 e741.

15. Yannuzzi LA, Wong DW, Sforzolini BS, et al. Polypoidal choroidal vasculopathy and neovascularized age-related macular degeneration. Arch Ophthalmol. 1999;117(11):1503-1510.

16. Rouvas AA, Papakostas TD, Ntouraki A, Douvali M, Vergados I, Ladas ID. Angiographic and OCT features of retinal angiomatous proliferation. Eye (Lond). 2010;24(11):1633-1642; quiz 1643. 
17. Inoue R, Sawa M, Tsujikawa M, Gomi F. Association between the efficacy of photodynamic therapy and indocyanine green angiography findings for central serous chorioretinopathy. Am J Ophthalmol. 2010;149(3):441-446 e441-442.

18. Yannuzzi LA, Slakter JS, Gross NE, et al. Indocyanine green angiography-guided photodynamic therapy for treatment of chronic central serous chorioretinopathy: a pilot study. Retina. 2003;23(3):288-298.

19. Bouchenaki N, Cimino L, Auer C, Tao Tran V, Herbort CP. Assessment and classification of choroidal vasculitis in posterior uveitis using indocyanine green angiography. Klin Monbl Augenheilkd. 2002;219(4):243-249.

20. Balasubramaniam SC, Pellegrini M, Staurenghi G, Pulido JS. Infrared Imaging of Circumscribed Choroidal Hemangiomas. Retina. 2017;37(6):1134-1139.

21. Schalenbourg A, Piguet B, Zografos L. Indocyanine green angiographic findings in choroidal hemangiomas: A study of 75 cases. Ophthalmologica. 2000;214(4):246-252.

22. Shields JA, Mashayekhi A, Ra S, Shields CL. Pseudomelanomas of the posterior uveal tract: the 2006 Taylor R. Smith Lecture. Retina. 2005;25(6):767-771.

23. Shields CL, Shields JA, De Potter P. Patterns of indocyanine green videoangiography of choroidal tumours. Br J Ophthalmol. 1995;79(3):237-245.

24. Shiraki K, Moriwaki M, Yanagihara N, Kohno T, Miki T. Indocyanine green angiograms of choroidal nevi. comparison between confocal and nonconfocal scanning laser ophthalmoscope and fundus video camera. Jpn J Ophthalmol. 2001;45(4):368-374.

25. Tan CS, Ting DS, Lim LW. Multicolor Fundus Imaging of Polypoidal Choroidal Vasculopathy. Ophthalmol Retina. 2019;3(5):400-409.

26. Campagnoli TR, Medina CA, Singh AD. Choroidal Melanoma Initially Treated as Hemangioma: Diagnostic and Therapeutic Considerations. Retin Cases Brief Rep. 2016;10(2):175-182.

27. Al-Dahmash SA, Shields CL, Kaliki S, Johnson T, Shields JA. Enhanced depth imaging optical coherence tomography of choroidal metastasis in 14 eyes. Retina. 2014;34(8):1588-1593.

28. Aronow ME, Portell CA, Sweetenham JW, Singh AD. Uveal lymphoma: clinical features, diagnostic studies, treatment selection, and outcomes. Ophthalmology. 2014;121(1):334-341.

29. Shields CL, Manalac J, Das C, Saktanasate J, Shields JA. Review of spectral domain enhanced depth imaging optical coherence tomography of tumors of the choroid. Indian $J$ Ophthalmol. 2015;63(2):117-121.

30. Witschel H, Font RL. Hemangioma of the choroid. A clinicopathologic study of 71 cases and a review of the literature. Surv Ophthalmol. 1976;20(6):415-431.

31. Slakter JS, Yannuzzi LA, Sorenson JA, Guyer DR, Ho AC, Orlock DA. A pilot study of indocyanine green videoangiography-guided laser photocoagulation of occult choroidal neovascularization in age-related macular degeneration. Arch Ophthalmol. 1994;112(4):465-472.

32. Shields CL, Dalvin LA, Lim LS, et al. Circumscribed Choroidal Hemangioma: Visual Outcome in the Pre-Photodynamic Therapy Era versus Photodynamic Therapy Era in 458 Cases. Ophthalmol Retina. 2019.

33. Papastefanou VP, Plowman PN, Reich E, et al. Analysis of Long-term Outcomes of Radiotherapy and Verteporfin Photodynamic Therapy for Circumscribed Choroidal Hemangioma. Ophthalmol Retina. 2018;2(8):842-857. 


\section{FIGURES:}

Figure 1. (A) Digital colour photograph depicting a red-orange $\mathrm{CCH}$ superior to the optic nerve in the right eye. (B) Fine, lacy intrinsic vessels are appreciated on the early DCS ICG frames; (C) however by the time maximal hyperfuorescence is reached the intrinsic vascular architecture of the lesion can no longer be appreciated and the $\mathrm{CCH}$ takes on a diffuse, feathery pattern of hyperfluorescence. (D) At the latest frame, there is partial washout of the lesion (white stars) and an early hyperfluorescent rim (black stars).

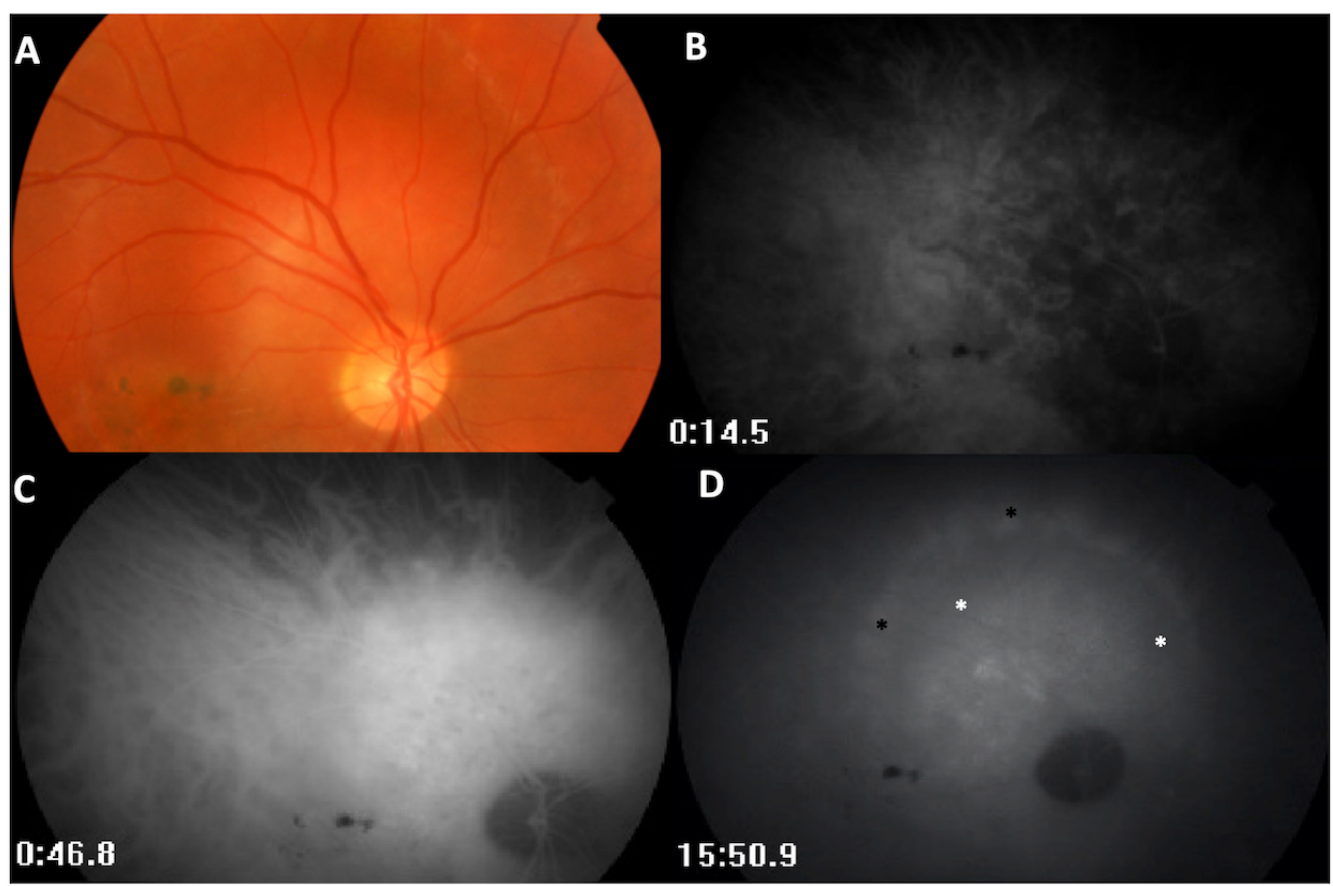


Figure 2. (A) Digital colour photograph depicting a small, red-orange $\mathrm{CCH}$ superotemporal to the optic nerve in the right eye. (B) A few feathery intrinsic vessels are noted on early ICG frames (white arrows). (C) The lesion fills very rapidly, and appears diffusely hyperfluorescent with feathery borders obscuring the previously evident intralesional vascular architecture. (D) At the latest frame, there is a relative decrease in hyperfluorescence; however, no washout or hyperfluorescent rim is noted.

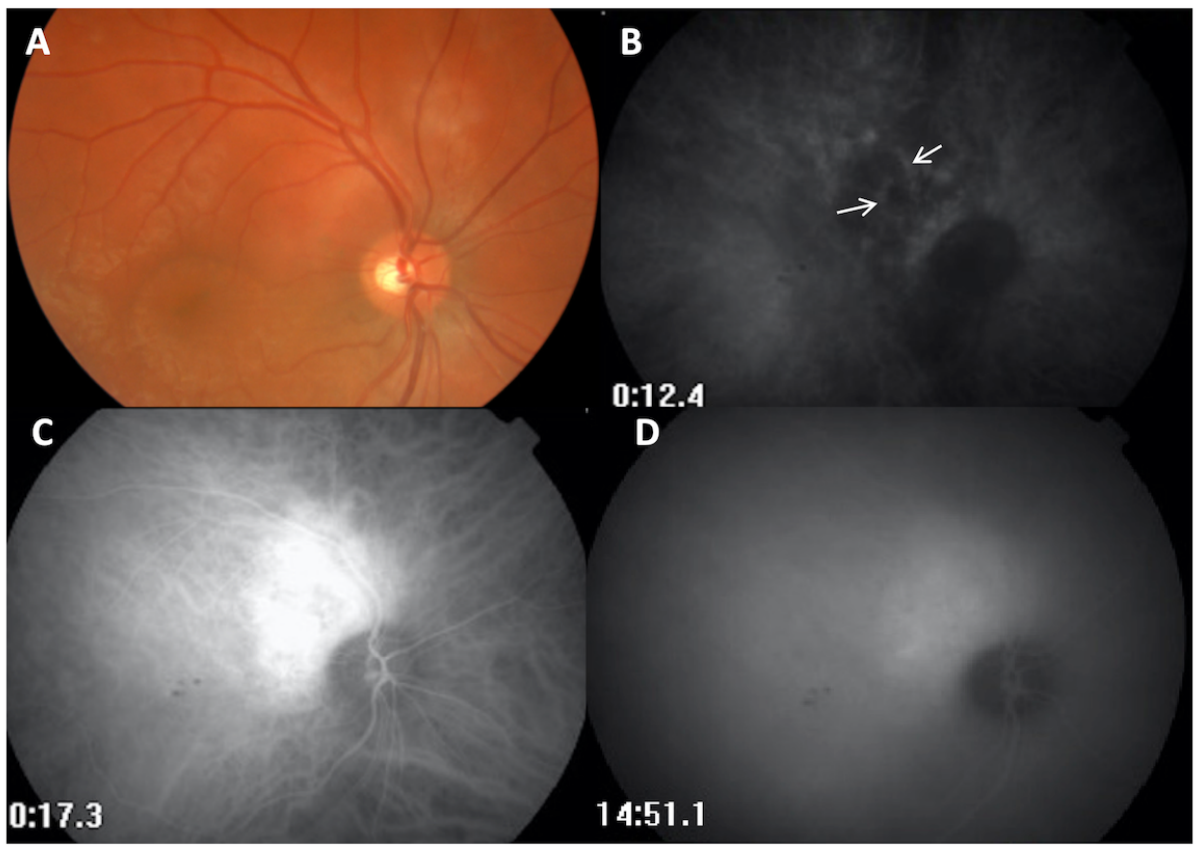


Figure 3. (A) Scanning laser ophthalmoscope (Optomap $\left.{ }^{\circledR}\right)$ pseudo-colour image of a superotemporal $\mathrm{CCH}$. (B) Red separation and (C) green separation images highlighting the choroidal location of the lesion. (D) Early SLO ICG (Spectralis) frames demonstrate prominent, lacy intrinsic vessels. (E) At maximum hyperfluorescence, a granular pattern of hyperfluorescence with several "hot spots" and visualization of vascular loops (black arrows). (F) On the latest frame, the $\mathrm{CCH}$ maintains a granular appearance with a number of hot spots remaining with partial washout (white star) and hyperfluorescent rim (black star).

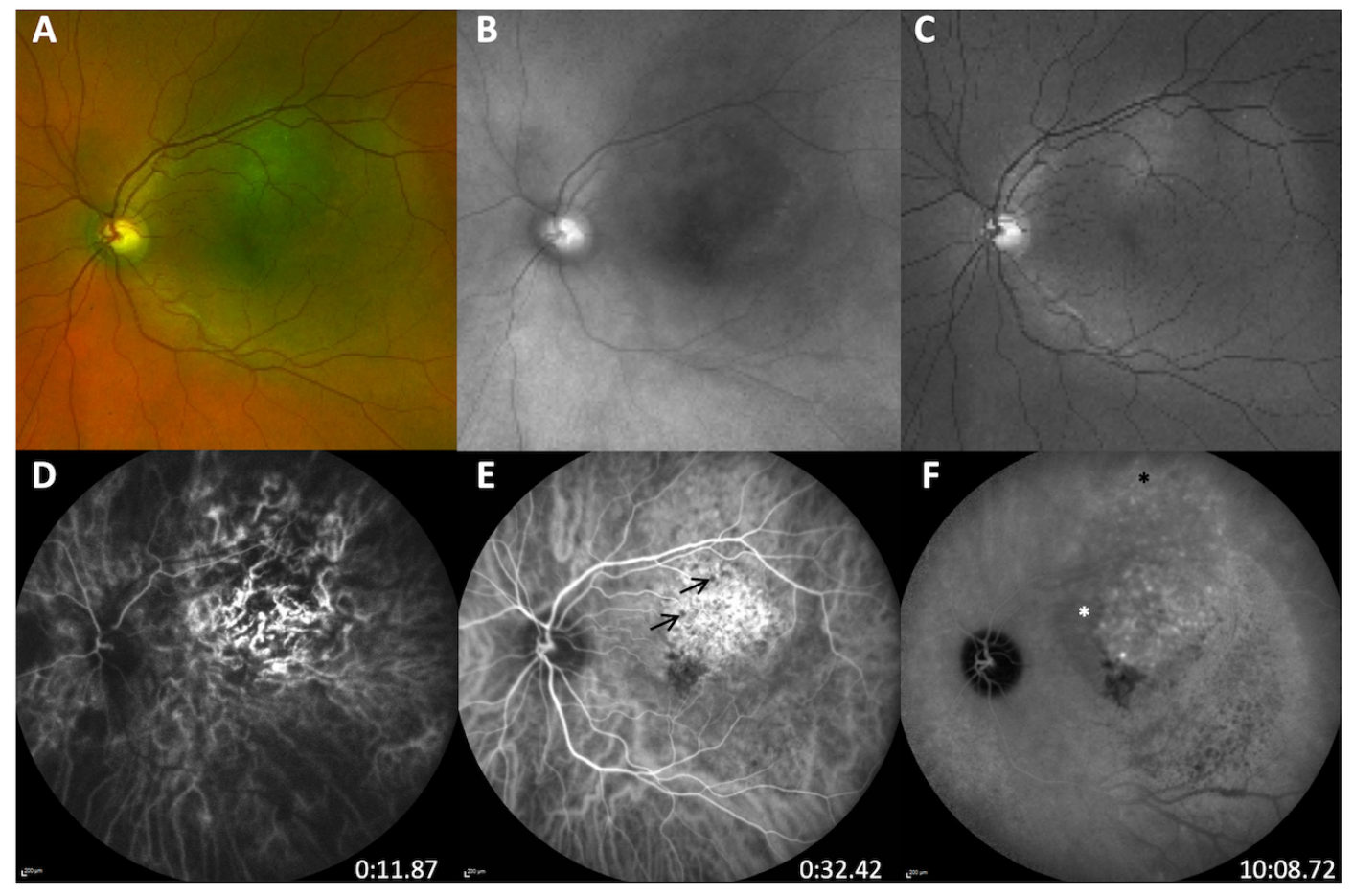


Figure 4. (A) Scanning laser ophthalmoscope (Optomap $\left.{ }^{\circledR}\right)$ pseudo-colour image of CCH with (B) red and (C) green separation images highlighting choroidal location of the lesion. (D) Prominent, lacy intrinsic vessels are well visualized on early Spectralis ICG frames and (E) at maximum hyperfluorescence, a granular pattern of hyperfluorescence with several "hot spots" and visualization of vascular loops (black arrow) is again apparent. (F) On the latest frame, there is partial washout (white stars). The vascular loops remain visualized into the latest frame (red arrows) and a partial hyperfluorescent rim (black star) can also be appreciated.

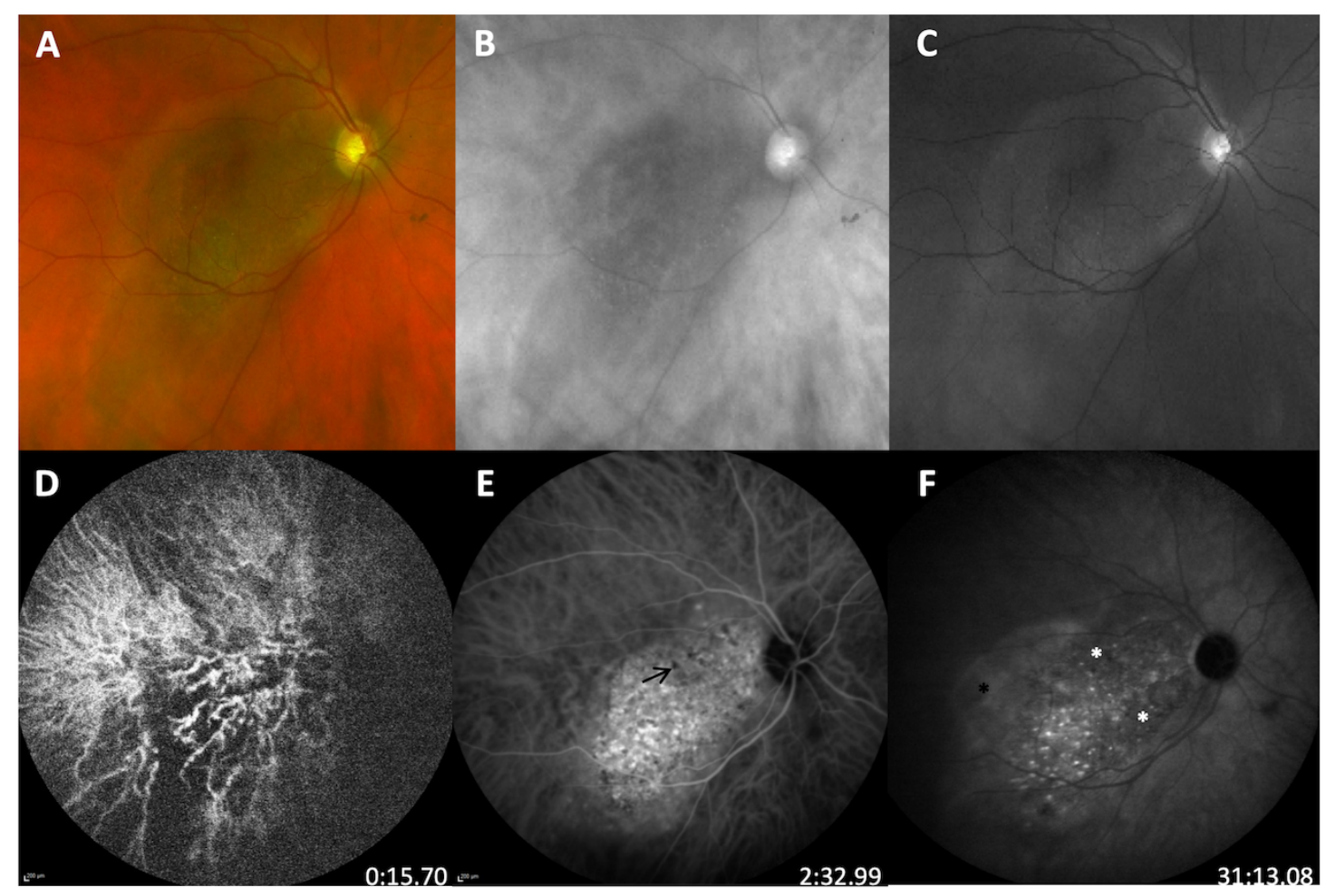

